\title{
Emergency Department Laparotomy Can Be a Resuscitative Option for Patient with Cardiac Arrest and Impending Arrest due to Intra-Abdominal Hemorrhage
}

\author{
Chan Ik Park a, Jae Hun Kim a, ${ }^{*}$, Kang Ho Lee a, Dong Yeon Ryu a, Hyun-Woo Sun a, Gil Hwan Kim a, Sang Bong Lee a, \\ Sung Jin Park a, Hohyun Kim a, Seok Ran Yeom ${ }^{b}$ \\ a Department of Trauma and Surgical Critical Care, Pusan National University Hospital, Busan, Korea

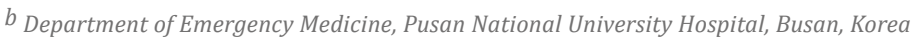

\section{Article history:}

Received: August 12, 2020

Revised: September 25, 2020

Accepted: October 14, 2020

\section{*Corresponding Author:}

Jae Hun Kim

Department of Trauma and Surgical Critical Care, Pusan National University Hospital 179 Gudeok-Ro, Seo-Gu, Busan, Korea E-mail:drtrauma73@gmail.com

\section{ORCID}

Chan Ik Park

https://orcid.org/0000-0001-8788-2381 Jae Hun Kim

https://orcid.org/0000-0003-4504-9898 Kang Ho Lee

https://orcid.org/0000-0003-4089-8831

Dong Yeon Ryu

https://orcid.org/0000-0003-2832-9572

Hyun-Woo Sun

https://orcid.org/0000-0003-0585-9538 Gil Hwan Kim

https://orcid.org/0000-0002-5449-5681

Sang Bong Lee

https://orcid.org/0000-0001-9396-1000

Sung Jin Park

https://orcid.org/0000-0002-4852-5442 Hohyun Kim

https://orcid.org/0000-0001-9434-8654

Seok Ran Yeom

https://orcid.org/0000-0002-1000-9637

\section{ABSTRACT}

Purpose: Managing patients with hemorrhagic shock is mainly dependent on stopping the bleeding as fast as possible. Emergency Department laparotomy (EDL) is considered one of the approaches to control intra-abdominal bleeding rapidly. This study aims to evaluate the outcomes of EDL in a regional trauma center of Pusan National University Hospital in a 4-year period.

Methods: The medical records and data of patients who underwent EDL from January 2016 to December 2019 were analyzed. Patients who underwent preperitoneal pelvic packing only or did not receive surgery immediately after EDL were excluded.

Results: Twenty-four patients who underwent EDL were included in the study. 18 patients had sustained blunt trauma, and 6 suffered from penetrating injuries. Small bowel mesentery and liver injuries were the most frequent. Increase of median systolic blood pressure (SBP) after EDL was 55.5 $\mathrm{mmHg}$. Four (16.7\%) out of the 24 survived; one of the four survivors received cardiopulmonary resuscitation (CPR). In the nonsurvivor group, Injury Severity Score was significantly higher $(p=$ $0.013)$, initial $\mathrm{pH}$ was lower $(p=0.035)$ and the amount of packed red blood cells transfusion after EDL was significantly higher $(p=0.013)$ than those in the survivor group.

Conclusion: The mortality rate was very high in trauma patients who were required EDL. Although EDL was not proved to be an effective procedure for resuscitation in trauma patients, it could be considered as one of the treatment options for trauma patients in extremis. Further studies are required to examine the effects of EDL.

Keywords: emergency department, laparotomy, hemorrhage, resuscitation

\section{Introduction}

Noncompressible torso hemorrhage is one of the leading causes of trauma-related death, posing a great challenge for trauma surgeons. The faster bleeding is stopped, the more likely the patient will survive [1]. To control noncompressible torso hemorrhage, many challenges have been doing. One of the most popular and effective methods to control noncompressible torso hemorrhage is resuscitative endovascular balloon occlusion of aorta (REBOA) [2-4]. However, this approach has its disadvantages, such as procedure failure which could be as high as $19-40 \%[5,6]$. Vascular access can result in unnecessary complications in patients who require surgery to stop the bleeding. A prolonged occlusion period could lead to ischemia 
and reperfusion injury of the distal organs [7].

Emergency department laparotomy (EDL) as a resuscitative procedure could solve the above-mentioned shortcomings. Several studies were conducted on performing EDL in hemodynamically unstable patients due to intra-abdominal injuries; although the mortality rate was high, but it seemed to have a resuscitative effect [8-10]. In our institute, hemodynamically unstable patients due to intra-abdominal injury were selected for EDL.

The purpose of this study is to assess the effects of EDL as a resuscitative treatment.

\section{Materials and Methods}

A retrospective review of the medical records of the patients who received EDL in a trauma emergency room in a regional trauma center of Pusan National University Hospital was carried out. Exclusion criteria were as follows: patients who underwent preperitoneal pelvic packing only; did not received surgery immediately after EDL. The following variables were analyzed: patients' demographics, injury type, Injury Severity Score (ISS), Abbreviated Injury Score (AIS) of the abdominal region, initial $\mathrm{pH}$, lactate level, hemoglobin, platelet count, prothrombin time (PT) (international normalized ratio, INR), fibrinogen level, organs and vessels addressed in EDL, systolic blood pressure (SBP) before and after EDL, aortic crossclamping, cardiopulmonary resuscitation (CPR), the amount of packed red blood cells (pRBC) transfused before and after EDL, and time of death.

EDL was performed in a patient with an intra-abdominal fluid collection detected on focused assessment with sonography

Table 1. Indications and contraindications of EDL of Pusan National University Hospital.

\section{Indications}

A. After resuscitation, non-responder (systolic blood pressure $<70$ $\mathrm{mmHg}$ )

B. A positive result on focused assessment with sonography for trauma

C. Level of consciousness: stupor or lower

Contraindications

The patient showing no signs of life

* Signs of life:

a. Cardiac electrical activity

b. Pupillary response

c. Spontaneous ventilation

d. Extremity movement

$\mathrm{EDL}=$ Emergency Department laparotomy for trauma, where SBP was below $70 \mathrm{mmHg}$, showing no response after resuscitation with 1 liter of crystalloid fluid and 2 units of pRBC. Indication and contraindications of EDL of Pusan National University Hospital are mentioned in Table 1. The trauma emergency room was equipped with two sets of instruments and laparotomy pads in preparation for EDL; the contents of these sets are displayed in Table 2.

For selected patients, endotracheal intubation was performed and continuous remifentanil infusion and intravenous muscle relaxant bolus injection were administered. The patients' skin was sterilized with betadine solution and fully covered with aseptic surgical drapes. Subsequently, long midline incision was made and bleeding was controlled by four-quadrant gauze packing and/or clamping of bleeding vessels until temporary hemostasis was achieved. Aortic cross clamping was performed selectively depending on discretion of the surgeon and the patient's hemodynamic status. Thereafter, temporary abdominal closure with adhesive antimicrobial incise drapes was conducted; then, the patients were taken to the operating room for damage control surgery.

The primary outcome was width of SBP change after EDL compared to before EDL. The secondary outcome was inhospital mortality.

Table 2. List of the contents in the instrument sets for EDL.

\begin{tabular}{|c|c|}
\hline Instrument & Number \\
\hline Needle holder & 2 \\
\hline Aortic cross clamp & 1 \\
\hline 25 cm Kelly clamp, curved & 1 \\
\hline 20 cm Kelly clamp, curved & 5 \\
\hline $15 \mathrm{~cm}$ Kelly clamp, curved & 5 \\
\hline Mosquito clamp, curved & 5 \\
\hline Right angle clamp & 2 \\
\hline Richardson retractor & 2 \\
\hline Mayo scissors & 1 \\
\hline Metzenbaum scissors & 1 \\
\hline Long pincette & 1 \\
\hline DeBakey tissue forceps & 2 \\
\hline Tissue forceps $1-2$ teeth & 1 \\
\hline Poole abdominal suction tip & 1 \\
\hline Yankauer suction tube & 1 \\
\hline Scalpel handle & 1 \\
\hline Sponge forceps & 2 \\
\hline Towel clamp & 2 \\
\hline Anatomical tissue forceps & 1 \\
\hline
\end{tabular}

$\mathrm{EDL}=$ Emergency Department laparotomy. 
This study was approved by the Institutional Review Board of Pusan National University Hospital (no.: H-2007-001-092).

Fisher's exact test and Mann-Whitney U test were employed to analyze clinical data, where appropriate. A two-sided $\mathrm{p}$ value less than 0.05 was considered to be statistically significant. Statistical analyses were carried out using SPSS, Version 22.0, for Windows (IBM Corp., Armonk, NY, USA)

\section{Results}

From January 2016 to December 2019, twenty-five patients underwent EDL in the trauma emergency room of our regional trauma center. One patient was excluded because of insufficient data in the medical records about injured organs or vessels in EDL; therefore, only twenty-four cases were included in this study.

19 patients were male (79.2\%) and the median age was 53.5 years (range: $22-75$ ). 18 patients were injured by blunt trauma (75\%) and 6 were injured by penetrating trauma (25\%). The median of ISS was 29 (range: 16-50). The median of maximum AIS of abdominal region was 4 (range: $3-5$ ). ISS was significantly higher in the nonsurvivor group than in the survivor group ( $p=0.013$ ). In the nonsurvivor group, the initial $\mathrm{pH}$ was significantly lower than the survivor group ( $p=0.035)$; however, there was no statistically significant differences regarding the other variables between the survivor and the nonsurvivor groups (Table 3 ).

Injured organs and vessels were mesentery, liver, spleen, small bowel, colon, pancreas, iliac artery or vein, kidney, duodenum, and stomach. Mesentery and liver were the most frequent injured sites in EDL, as shown in Table 4.

24 patients underwent EDL. There were 4 survivors (survivor group) and 20 death (nonsurvivor group). In-hospital mortality rate was $83.3 \%$ ( 20 of 24 ). We evaluated the difference according to clinical variables between two groups. The median SBP before EDL was $37.5 \mathrm{mmHg}$ and after EDL was $90 \mathrm{mmHg}$. The median SBP after EDL increased by $55.5 \mathrm{mmHg}$. There was no significant difference in SBP before and after EDL between the survivor and nonsurvivor groups. Six patients underwent aortic cross-clamping, and all of them died. 15 patients received CPR; only one patient survived. The median duration

Table 4. The injured organs and vessels that were diagnosed in EDLs ( $n$ = 24).

\begin{tabular}{|ll|}
\hline Organ or vessel & $n$ \\
\hline Mesentery & 12 \\
Liver & 12 \\
\hline Spleen & 10 \\
\hline Small bowel & 7 \\
\hline Colon & 4 \\
\hline Pancreas & 3 \\
\hline Iliac artery or vein & 2 \\
\hline Kidney & 2 \\
\hline Duodenum & 2 \\
\hline Stomach & 1 \\
\hline
\end{tabular}

Table 3. Patient characteristics and initial laboratory findings in trauma emergency room.

\begin{tabular}{|c|c|c|c|c|}
\hline Variables & $\begin{array}{c}\text { Total } \\
(n=24)\end{array}$ & $\begin{array}{l}\text { Survivor } \\
(n=4)\end{array}$ & $\begin{array}{l}\text { Nonsurvivor } \\
\quad(n=20)\end{array}$ & $p$ \\
\hline Sex, M:F & 19:5 & $2: 2$ & $17: 3$ & 0.179 \\
\hline Age (median) & 53.5 & 54 & 53.5 & 0.852 \\
\hline \multicolumn{5}{|l|}{ Type of injury } \\
\hline Blunt : penetrating & 18:6 & $3: 1$ & $15: 5$ & 0.749 \\
\hline ISS (median) & 29 & 22 & 33 & 0.013 \\
\hline Maximum AIS in the abdomen region (median) & 4 & 3.5 & 4 & 0.135 \\
\hline Initial pH (median) & 7.06 & 7.32 & 7.01 & 0.035 \\
\hline Initial lactate, $\mathrm{mmol} / \mathrm{L}$ (median) & 12.3 & 7.65 & 12.8 & 0.188 \\
\hline Initial hemoglobin, $\mathrm{g} / \mathrm{dL}$ (median) & 9.3 & 5.7 & 10.5 & 0.054 \\
\hline Initial platelet count, $103 / \mu \mathrm{L}$ (median) & 137 & 135 & 168 & 0.366 \\
\hline Initial prothrombin time (INR ; median) & 1.71 & 1.39 & 1.75 & 0.138 \\
\hline Initial fibrinogen, mg/dL (median) & 93.1 & 100.1 & 93.1 & 0.635 \\
\hline
\end{tabular}

TER = trauma emergency room; ISS = injury severity score; AIS = abbreviated injury scale; INR = international normalized ratio. 
Table 5. Results of treatment for the patients who underwent EDL.

\begin{tabular}{|c|c|c|c|c|}
\hline Variables & $\operatorname{Total}(n=24)$ & Survivor $(n=4)$ & Nonsurvivor $(n=20)$ & $p$ \\
\hline SBP before EDL, mmHg (median) & 37.5 & 50 & 33.5 & 0.477 \\
\hline SBP after EDL, mmHg (median) & 90 & 84 & 90 & 0.991 \\
\hline Difference of SBP between before and after EDL, mmHg (after - before) & 55.5 & 53.5 & 57 & 0.794 \\
\hline Done of aortic cross clamping & 6 & $0(0 \%)$ & $6(100 \%)$ & 0.539 \\
\hline Done of CPR & 15 & $1(6.7 \%)$ & $14(93.3 \%)$ & 0.13 \\
\hline Duration of CPR, total, minutes (median) & 14 & 3 & 15 & 0.4 \\
\hline From admission to EDL, minutes (mean $\pm \mathrm{SD}$ ) & $29.6 \pm 17.7$ & $24.0 \pm 11.9$ & $33.6 \pm 18.6$ & 0.682 \\
\hline Duration of EDL, minutes (mean $\pm \mathrm{SD}$ ) & $11.7 \pm 6.4$ & $7.5 \pm 2.9$ & $12.5 \pm 6.6$ & 0.135 \\
\hline From EDL to operation room, minutes (mean \pm SD) & $17.7 \pm 13.3$ & $14.0 \pm 10.2$ & $18.4 \pm 14.0$ & 0.667 \\
\hline Packed RBC transfusion before EDL, unit (mean \pm SD) & $2.7 \pm 1.5$ & $2.8 \pm 1.5$ & $2.7 \pm 1.6$ & 0.97 \\
\hline Packed RBC transfusion after EDL, unit (mean \pm SD) & $39.0 \pm 27.9$ & $14.5 \pm 4.7$ & $43.9 \pm 28.1$ & 0.013 \\
\hline
\end{tabular}

$\mathrm{SBP}=$ systolic blood pressure; $\mathrm{EDL}=$ emergency department laparotomy; $\mathrm{CPR}=$ cardiopulmonary resuscitation; $\mathrm{RBC}=$ red blood cell; $\mathrm{EDL}=$ Emergency Department laparotomy.

Table 6. Time from admission to death.

\begin{tabular}{lc}
\hline Time from admission to death & Number of patients $(n=20)$ \\
$<24$ hours & $12(60)$ \\
$24-48$ hours & $3(15)$ \\
$>48$ hours & $5(25)$ \\
\hline
\end{tabular}

Data are presented as $n$ (\%).

of CPR was 3 minutes in survivor group and 15 minutes (range 2-49 minutes) in the nonsurvivor group. The duration of CPR in nonsurvivor group tended to be longer than in survivor group, but there was no statistical difference. Time from admission to EDL was $29.6 \pm 17.7$ minutes (mean \pm SD), duration of EDL was $11.7 \pm 6.4$ minutes (mean $\pm \mathrm{SD}$ ), and time from EDL to operation room was $17.7 \pm 13.3$ minutes (mean \pm SD). There were no significant differences according to the above three variables between two groups. The amount of pRBC transfusion before EDL was $2.8 \pm 1.5$ units (mean $\pm \mathrm{SD}$ ) in survivor group and $2.7 \pm 1.6$ units (mean \pm SD) in nonsurviror group. There was no significant difference between two groups. The amount of pRBC transfusion after EDL was $14.5 \pm 4.7$ units (mean \pm $\mathrm{SD}$ ) in survivor group and $43.9 \pm 28.1$ units (mean \pm SD) in nonsurvivor group. In nonsurvivor group, the amount of pRBC transfusion after EDL was significantly higher than survivor group $(p=0.013)$. Details were shown on Table 5 .

We evaluate the time from admission to death in nonsurvivor group $(n=20) .12$ patients $(60 \%)$ died within 24 hours from admission. Five patients (25\%) died over 48 hours from admission (Table 6).

\section{Discussion}

In 1979, Mattox et al [8] published a study on EDL and since then, some studies have been published in English literature [9-10]. However, the patients' characteristics in each study were somewhat different. The study of Mattox et al [8] was similar to this study in terms of using EDL as a resuscitative procedure rather than a definitive treatment. In the study of Mattox et al [8], 11 out of 51 patients were transferred to the operating room after EDL, but in-hospital mortality rate was $100 \%$. In this study, 23 of 24 patients with EDL were taken to the operating room and 12 patients died within 24 hours (Table 6). Finally, four patients survived. A simple comparison between two studies would not be relevant, because of different resuscitation technique, operation skill, and patients' characteristics. Further multicenter studies are required to examine the effects of EDL on mortality rate.

REBOA has recently become popular resuscitation procedure in hemodynamically unstable patients due to noncompressible torso hemorrhage [2-4]. REBOA can be performed under local anesthesia by accessing the femoral artery within a short time. However, this technique has some complications as follows. It is very challenging to access blood vessels in hemodynamically unstable patients while performing REBOA. Thus, vascular access failure could occur, as high as $40 \%$ [5]. Another major complication is organ ischemia below the occlusion level. To overcome this issue, partial occlusion was conducted during the REBOA procedure [11,12]. Compared to REBOA, EDL has advantage that perfusion of intact organs is preserved. Since, direct compression and clamping are performed on only damaged organs and blood vessels in EDL. In this study, blood 
pressure was restored in 18 out of 24 patients by applying gauze packing around the damaged organs and clamping of the damaged blood vessels to achieve temporary hemostasis in EDL. However, in six patients, blood pressure was not restored after EDL, aortic cross-clamping was required to maintain cerebral and coronary perfusion pressure; however, all of them died.

Infection can be an issue due to relatively unclean environment of emergency room compared with operating room. But, four survivors of this study had no EDL-related infection. There was one infection-related death in nonsurvivor group. However, EDL-related infection was not identified in this patient. The patient had lung contusion and rib fractures with liver injury. The patient developed pneumonia in the intensive care unit, and died of sepsis caused by pneumonia. Because the small number of patients were included, infection risk of EDL cannot be determined in this study. Further studies are required for evaluating the infection risk of EDL.

The ISS was higher in the nonsurvivor group than the survivor group (Table 3). Actually, real ISS of nonsurvivor group could be greater. In this study, three blunt trauma patients who died within 24 hours did not undergo any radiologic examination except chest X-ray. In case of patients who died within a short time without sufficient radiologic examination, ISS cannot be accurately determined. Autopsy is one of the ways that can find out unrevealed injury and its severity, but autopsy is uncommon in South Korea. If accurate examinations were made through autopsy or post-mortem imaging studies, the difference of ISS between two groups could be increased.

Traumatic cardiac arrest occurred in 15 among 24 patients. Four patients were victims of penetrating trauma and 11 were those of blunt trauma. 15 patients underwent CPR, and only one patient survived, who was victim of the penetrating injury and received CPR for 3 minutes. In this study, the survival rate of patients who underwent CPR was 6.7\% (1/15). It seems to be a relatively high survival rate compared to previous studies that reported a survival rate as from $2.0 \%$ to $7.5 \%$ [13-15].

Out of the 20 patients of nonsurvivors, 15 patients died within 48 hours from the admission as a result of uncontrolled hemorrhage and acute traumatic coagulopathy. Among 5 patients who died over 48 hours from admission, 1 patient died due to sepsis caused by pneumonia and 4 patients died due to multiorgan failure because of ischemia. Significant efforts must be made to minimize bleeding time and tissue ischemia by improving patient transport system in our region and treatment process in our trauma center.

The present study had several limitations as follows. First, this study was not a randomized, controlled trial, since it is challenging to carry out a randomized trial in an emergent setting, where there is very little time for a patient's legal representative to assess and give a consent for the randomized treatment. Second, the findings and statistical differences of this study cannot be generalized due to the small number of patients in a single institute. Third, this is a single cohort study for patients treated with EDL. Because there was no data for patients treated with other approaches such as REBOA in our institution, it has a limitation to compare EDL with other approaches.

Further studies are required to assess the resuscitative effect of EDL and its impact on mortality rate. A multicenter study comparing the EDL group and the control group using other treatment methods such as REBOA and using propensity score matching is required.

\section{Conclusion}

In this study, the mortality rate was very high in trauma patients who were required EDL. It seems that EDL could increase SBP of hemodynamically unstable patient with abdominal injuries temporarily, but resuscitative effect of EDL is not definitely identified. Therefore, EDL could be considered as one of the treatment options for trauma patients in extremis. Further multicenter studies are required to examine the effects of EDL on survival.

\section{Conflicts of Interest}

No potential conflict of interest relevant to this article was reported.

\section{Acknowledgement}

This work was supported by clinical research grant from Pusan National University Hospital in 2019.

\section{References}

[1] Chang R, Kerby JD, Kalkwarf KJ, Van Belle G, Fox EE, Cotton BA, et al. Earlier time to hemostasis is associated with decreased mortality and rate of complications: Results from the Pragmatic Randomized Optimal Platelet and Plasma Ratio trial. J Trauma Acute Care Surg 2019;87(2):3429.

[2] Kim DH, Chang SW, Matsumoto J. The utilization of resuscitative endovascular balloon occlusion of the aorta: preparation, technique, and the implementation of a novel approach to stabilizing hemorrhage. J Thorac Dis 2018;10(9):5550-9.

[3] Bekdache O, Paradis T, Shen YBH, Elbahrawy A, Grushka J, Deckelbaum D, et al. Resuscitative endovascular balloon occlusion of the aorta (REBOA): indications: advantages and challenges of implementation in traumatic non-compressible torso hemorrhage. Trauma Surg Acute Care Open 2019;4(1):e000262.

[4] Yamamoto R, Cestero RF, Suzuki M, Funabiki T, Sasaki J. Resuscitative endovascular balloon occlusion of the aorta (REBOA) is associated with 
improved survival in severely injured patients: A propensity score matching analysis. Am J Surg 2019;218(6):1162-8.

[5] Low RB, Longmore W, Rubinstein R, Flores L, Wolvek S. Preliminary report on the use of the Percluder occluding aortic balloon in human beings. Ann Emerg Med 1986;15(12):1466-9.

[6] Tsurukiri J, Akamine I, Sato T, Sakurai M, Okumura E, Moriya M, et al. Resuscitative endovascular balloon occlusion of the aorta for uncontrolled haemorrahgic shock as an adjunct to haemostatic procedures in the acute care setting. Scand J Trauma Resusc Emerg Med 2016;24:13.

[7] Markov NP, Percival TJ, Morrison JJ, Ross JD, Scott DJ, Spencer JR, et al. Physiologic tolerance of descending thoracic aortic balloon occlusion in a swine model of hemorrhagic shock. Surgery 2013;153(6):848-56.

[8] Mattox KL, Allen MK, Feliciano DV. Laparotomy in the emergency department. JACEP 1979;8(5):180-3.

[9] Lund H, Kofoed SC, Hillingsø JG, Falck-Larsen C, Svendsen LB. High mortality after emergency room laparotomy in haemodynamically unstable trauma patients. Dan Med Bull 2011;58(5):A4275.

[10] Ito K, Nakazawa K, Nagao T, Chiba H, Miyake Y, Sakamoto T, Fujita T. Emergency trauma laparotomy and/or thoracotomy in the emergency department: risks and benefits. Trauma Surg Acute Care Open 2019;4(1):e000269.

[11] Matsumura Y, Matsumoto J, Kondo H, Idoguchi K, Ishida T, Kon Y et al. Fewer REBOA complications with smaller devices and partial occlusion: evidence from a multicentre registry in Japan. Emerg Med J 2017;34(12):793-9.

[12] Sadeghi M, Hörer TM, Forsman D, Dogan EM, Jansson K, Kindler C, et al. Blood pressure targeting by partial REBOA is possible in severe hemorrhagic shock in pigs and produces less circulatory, metabolic and inflammatory sequelae than total REBOA. Injury 2018;49(12):2132-41.

[13] Georgescu V, Tudorache O, Strambu V. Traumatic cardiac arrest in the emergency department - Overview upon primary causes. J Med Life 2014;7(2):287-90.

[14] Deasy C, Bray J, Smith K, Harriss L, Morrison C, Bernard S, et al. Traumatic out-of-hospital cardiac arrests in Melbourne, Australia. Resuscitation 2012;83(4):465-70.

[15] Lockey D, Crewdson K, Davies G. Traumatic cardiac arrest: who are the survivors? Ann Emerg Med 2006;48(3):240-4. 